\title{
PARP-1 controls NK cell recruitment to the site of viral infection
}

\author{
Qiyang Shou, ${ }^{1,2}$ Huiying Fu, ${ }^{1,2}$ Xiaopei Huang, ${ }^{1}$ and Yiping Yang ${ }^{1,3}$ \\ 'Division of Hematologic Malignancies and Cellular Therapy, Department of Medicine, Duke University Medical Center, \\ Durham, North Carolina, USA. ${ }^{2}$ Second Clinical Medical College, Zhejiang Chinese Medical University, Hangzhou, China. \\ ${ }^{3}$ Department of Immunology, Duke University Medical Center, Durham, North Carolina, USA.
}

\begin{abstract}
The activation and recruitment of NK cells to the site of viral infection are crucial for virus control. However, it remains largely unknown what controls the recruitment of the activated NK cells to the infection site. In a model of intraperitoneal infection with vaccinia virus (VV), we showed that poly(ADP-ribose) polymerase-1 (PARP-1), a sensor of DNA damage, is critical for NK cell recruitment to the site of infection and viral control in vivo. We further demonstrated that PARP-1 promotes the production of CCL2 and that the CCL2-CCR2 axis is essential for NK cell recruitment to the infection site. In addition, we demonstrated that peritoneal macrophages are the main producer of PARP-1dependent CCL2 secretion. Mechanistically, PARP-1 functions as a regulator of NF- $\kappa B$ by promoting its nuclear translocation and binding to its response sequences in macrophages upon VV infection. Taken together, our results reveal a potentially previously unknown role for PARP-1-dependent CCL2 production in NK cell migration and viral control and may provide important insights into the design of effective NK cell-based therapies for viral infections and cancer.
\end{abstract}

\section{Authorship note: QS and HF contributed equally to this work. \\ Conflict of interest: The authors have declared that no conflict of interest exists.}

Copyright: (c) 2019, American Society for Clinical Investigation.

Submitted: March 23, 2018

Accepted: May 1, 2019

Published: June 20, 2019.

Reference information: /CI Insight. 2019;4(12):e121291. https://doi. org/10.1172/ji.insight.121291.

\section{Introduction}

NK cells play an important role in the control of various viral infections (1-3). Clinically, individuals with defective NK cell function are prone to severe and recurrent viral infections (4). NK cells are also critical for the control of vaccinia virus (VV), which is the most studied member of the poxvirus family and the live vaccine responsible for the successful elimination of smallpox (5). Previous studies have shown that NK cells are activated and recruited to the site of VV infection, leading to effective viral control (6-9). The activation of NK cells represents the initial step in the control of VV infection. We have further shown that efficient activation of NK cells is dependent on both TLR-dependent and TLR-independent pathways, as well as the NKG2D-activating receptor that recognizes host stress-induced NKG2D ligands (8-10). However, it remains largely unknown what controls the recruitment of the activated NK cells to the site of VV infection.

The chemokine receptors CCR2, CCR5, and CXCR3 have been implicated in NK cell recruitment to the site of viral infections in response to various chemokines (11). For example, CCR2 and CCR5 are both required for NK cell migration to the liver of murine cytomegalovirus-infected (MCMV-infected) mice $(12,13)$, whereas only CCR5 is needed for NK cell recruitment to the CNS in a model of herpes simplex virus (HSV) infection (14). On the other hand, NK cell migration to the liver of dengue virus-infected mice is dependent on CXCR3 (15). However, the role of chemokines and chemokine receptors in NK cell recruitment to the site of VV infection requires further investigation. Furthermore, their role remains undefined with regards to the molecular and cellular events following viral infection that trigger the secretion of chemokines and NK cell recruitment to the site of viral infection.

Poly(ADP-ribose) polymerases (PARPs) are a family of enzymes that catalyze the synthesis of ADP-ribose polymers using $\mathrm{NAD}^{+}$as a substrate and bind these branched polymers to acceptor proteins (16). The prototypical PARP enzyme, PARP-1, possesses a DNA-binding domain and becomes activated upon binding to damaged DNA (17). PARP-1 accounts for the majority of the poly(ADP-ribose) polymer synthesis and protein modifications near the DNA damage site. Thus, PARP-1 functions as a DNA damage sensor and is essential for DNA repair and genome stability (18). In addition, studies have shown that PARP-1 also plays an important role in the development of inflammation in a variety of models, including allergic encephalomyelitis (19), colitis (20), and septic shock $(21,22)$. More recently, PARP-1 has been implicated in the suppression of the number and function of Foxp3 ${ }^{+}$regulatory T cells, leading to enhanced proliferation of $\mathrm{CD}^{+} \mathrm{T}$ cells $(23,24)$. 
Given the dual roles of PARP-1 as a DNA damage sensor and a mediator of inflammation, we hypothesized that PARP-1 could sense DNA damage following viral infection and trigger the release of chemokines that recruit NK cells to the site of infection, leading to viral control. Indeed, here we provided evidence that although PARP-1 is not required for NK cell activation upon VV infection, it is critical for NK cell recruitment to the site of infection and viral control in vivo. We further showed that PARP-1 mediates the production of CCL2 and that NK cell migration is dependent on the CCL2-CCR2 axis. In addition, we demonstrated that peritoneal macrophages are the main producer of PARP-1-dependent CCL2 secretion. Furthermore, PARP-1 functions as a regulator of $\mathrm{NF}-\kappa \mathrm{B}$ by promoting its nuclear translocation and binding to its response sequences in macrophages upon VV infection. Collectively, these results highlight a critical role for PARP-1 in NK cell recruitment to the site of infection through NF- $\mathrm{KB}$-mediated production of CCL2 by macrophages in response to VV infection.

\section{Results}

PARP-1 is critical for NK cell recruitment to the site of VV infection and viral control. We first performed a timecourse experiment and showed that the NK cell recruitment to peritoneal cavity peaked at day 3 after intraperitoneal VV infection (Supplemental Figure 1; supplemental material available online with this article; https://doi.org/10.1172/jci.insight.121291DS1). To determine whether PARP-1 played a role in NK cell migration to the site of viral infection, mice were treated with different doses of the PARP-1 inhibitor AG14361 (25), followed by infection with VV, intraperitoneally. NK cell activation and migration to the peritoneal cavity and the viral load were assessed 3 days after infection. We identified that treatment with $10 \mathrm{mg} / \mathrm{kg}$ PARP-1 inhibitor in VV-infected mice led to a significant $(P<0.01)$ reduction in the total peritoneal NK cell numbers without obvious toxicity (Supplemental Figure 2), compared with VV-infected mice treated with PBS (Figure 1, A and B). However, IFN- $\gamma$ production by these NK cells was not affected (Figure 1C). Similarly, neither IFN- $\gamma$ production, nor in vitro NK cell killing function per NK cell basis was altered (data not shown). The reduction of NK cell recruitment to the peritoneal cavity by PARP-1 inhibition was accompanied by a significant $(P<0.01)$ increase in viral loads in the peritoneal cavity (Figure $1 D$ ). These results indicate that PARP-1 inhibition suppresses NK cell migration in response to intraperitoneal VV infection and delays viral clearance.

To further confirm the role of PARP-1 in NK cell migration, Parp- $1^{-/-}$mice were infected with VV intraperitoneally and examined for NK cell recruitment and the viral load in the peritoneal cavity. We showed that a significant $(P<0.01)$ reduction in the peritoneal NK cell numbers in $P a r p-1^{-/-}$mice, compared with WT mice (Figure 2, A and B), lead to a significant $(P<0.01)$ increase in viral loads in the peritoneal cavity (Figure 2D). Again, lack of PARP-1 did not affect the production of IFN- $\gamma$ by the Parp- $1^{-/-}$NK cells (Figure 2C). Collectively, these observations support a critical role of PARP-1 in NK cell recruitment to the site of VV infection and viral control.

PARP-1 mediates the production of CCL2 in response to VV infection. How does PARP-1 regulate NK cell migration to the site of VV infection? Since chemokines and chemokine receptors have been implicated in NK cell migration in other models of viral infection (12-15), we asked whether PARP-1 regulates the production of various chemokines in the peritoneal cavity. To address this question, cells from the peritoneal cavity were collected 12 hours after VV infection and assayed for CCL2, CCL3, CCL4, CCL5, CCL7, and CCL8 mRNA levels by real-time quantitative PCR. Our results showed that VV infection induced the expression of several chemokines, including CCL2, CCL3, and CCL4, compared with the naive uninfected controls (Figure 3A). Although a significant reduction in mRNA levels was found for CCL2, CCL3, and CCL4 in cells from VV-infected Parp- $1^{-/-}$mice compared with their WT counterparts, CCL2 expression in Parp-1/-1- mice demonstrated the greatest degree of reduction compared with that of WT counterparts (approximately 18-fold; Figure 3A). Indeed, the production of CCL2 protein was significantly diminished in Parp-1 ${ }^{-1-}$ mice (Figure 3B), suggesting PARP-1 controls CCL2 production in response to $\mathrm{VV}$ infection.

CCL2-CCR2 is responsible for NK cell migration to the site of VV infection. The observation that PARP-1 is critical for CCL2 production at the site of VV infection prompted us to examine whether CCL2 mediates NK cell migration in response to VV infection. We first examined whether the peritoneal fluid from the VV-infected mice mediated NK cell migration in an in vitro migration assay. Indeed, a marked increase in the number of NK cells migrated toward the VV-infected peritoneal fluid compared with uninfected controls (Figure 4A). The NK cell migration was significantly reduced when neutralizing anti-CCL2 
A
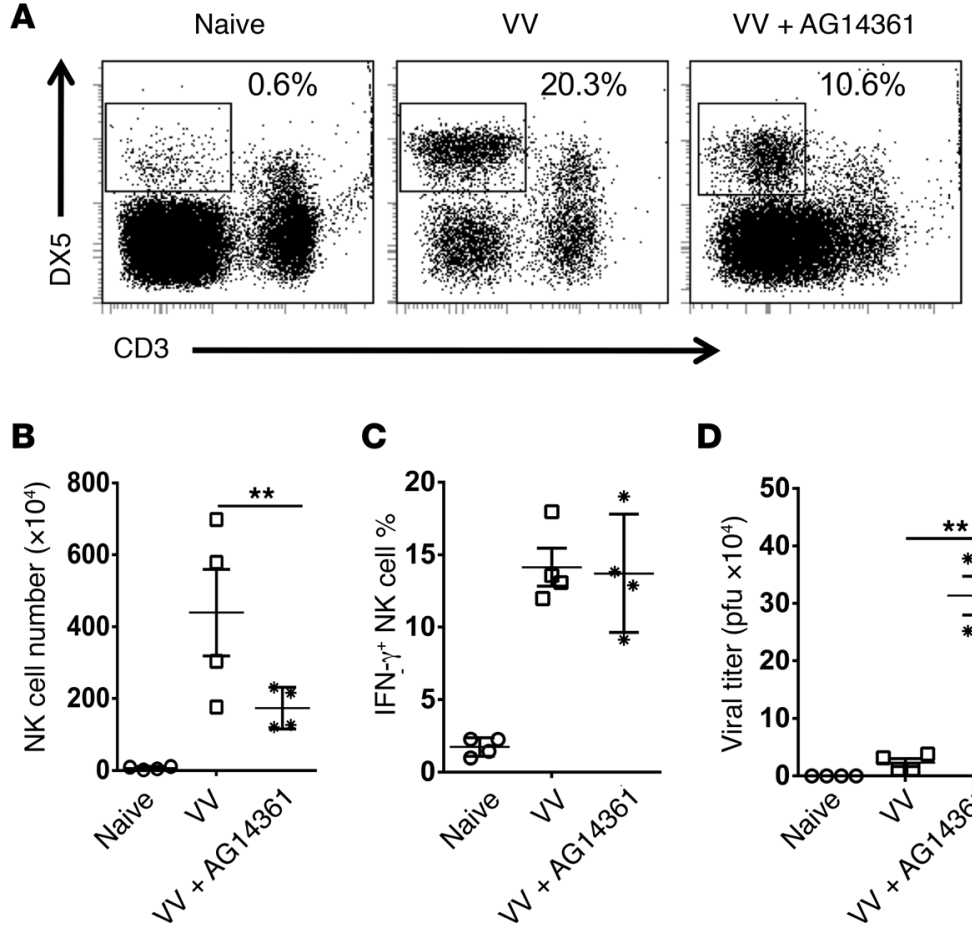

C

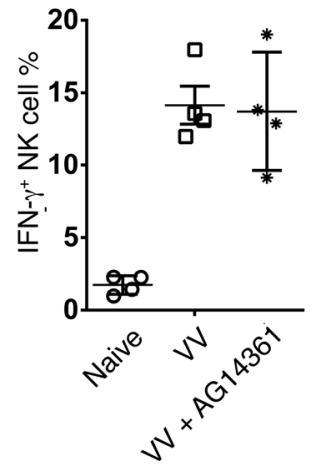

D

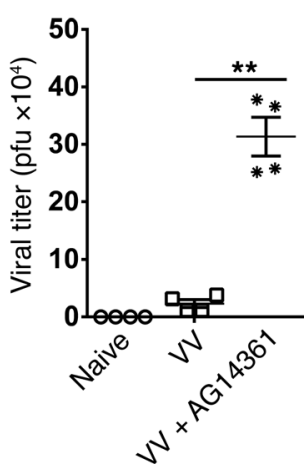

Figure 1. Inhibition of PARP-1 activity suppresses NK cell migration in response to intraperitoneal VV infection and delays viral clearance. C57BL/6 mice were either treated with PARP-1 inhibitor AG14361 (10 mg/kg, i.v.) (VV+AG14361) or PBS (VV) and subjected to infection with VV $\left(5 \times 10^{6} \mathrm{PFU}\right.$, i.p.). Naive mice were used as controls. Three days later, peritoneal fluid was harvested and assayed for DX5 $5^{+} \mathrm{CD} 3^{-}$NK cells by flow cytometry. (A) FACS plots showing percentages of DX5 ${ }^{+} \mathrm{CD}^{-}$NK cells in the peritoneal fluids. (B) The mean absolute NK cell numbers \pm SD are shown $(n=3)$. (C) IFN- $\gamma$ production was measured intracellularly and the mean percentages of IFN- $\gamma^{+}$NK cells are shown $(n=3)$. (D) The viral titer in peritoneal fluid was measured by the standard plaque assay and the mean viral titers \pm SD are shown $(n=$ 4). ${ }^{* *} P<0.01,2$-tailed Student's $t$ test.

antibodies were added to the peritoneal fluid (Figure 4A), suggesting that the NK cell migration is mediated by CCL 2 in vitro. We next examined whether NK cell migration in vivo is also dependent on CCL2. To address this question, mice were treated with anti-CCL2-neutralizing antibodies followed by VV infection intraperitoneally. We found that the anti-CCL2 antibody treatment significantly inhibited the NK cell recruitment in the peritoneal cavity (Figure 4B), supporting that CCL2 also mediates NK cell migration toward the site of viral infection in vivo.

To further support the role of CCL2 in mediating NK cell migration, we examined whether the CCL2 receptor CCR2 is also critical for NK cell migration. Indeed, NK cell recruitment to the peritoneal cavity was significantly reduced in $\mathrm{Ccr}^{-/-}$mice in response to VV infection, compared with the WT controls (Figure 5, A and B), leading to a significant increase in viral loads (Figure 5C). Taken together, these results indicate that NK cell recruitment to the site of VV infection is mediated by CCL2-CCR2.

Peritoneal macrophages are mainly responsible for PARP-1-dependent CCL2 production. We next examined which cell types are responsible for CCL2 production in response to VV infection in vivo. Mice were infected with VV intraperitoneally, and CCL2 production in NK, T, and B cells and macrophages within the peritoneal cavity, was measured by intracellular staining. Our results showed that peritoneal macrophages are the main producers of CCL2 (Figure 6A). We further found that the production of CCL2 by peritoneal macrophages was significantly diminished in Parp-1 ${ }^{-1-}$ mice (Figure 6, B and C). These results suggest that macrophages in peritoneal cavity are mainly responsible for PARP-1-dependent CCL2 production.

PARP-1 promotes NF- $\kappa B$ activation in peritoneal microphages. We next investigated how PARP-1 mediates CCL2 production in macrophages upon VV infection. Since studies have shown that the production of inflammatory cytokines, such as IL- 6 and TNF- $\alpha$, is mediated by NF- $\kappa$ B (26), and these cytokines boost the expression of other cytokines, chemokines, and adhesion molecules to promote the inflammatory processes (27). Thus, we asked if PARP-1 promotes the activation of NF- $\mathrm{KB}$ in peritoneal 
A

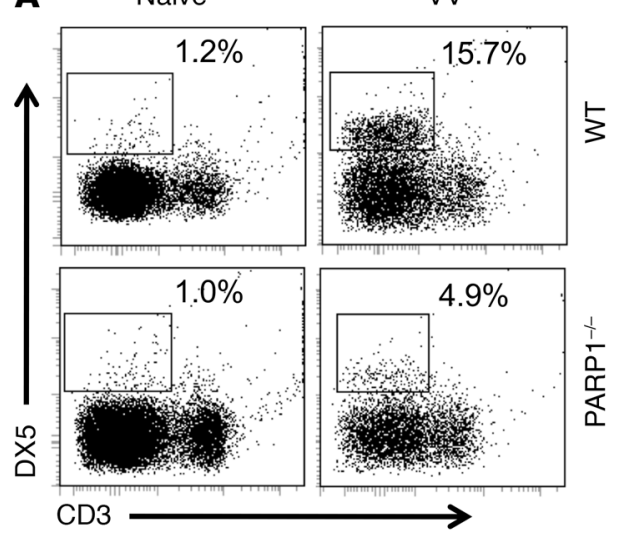

C

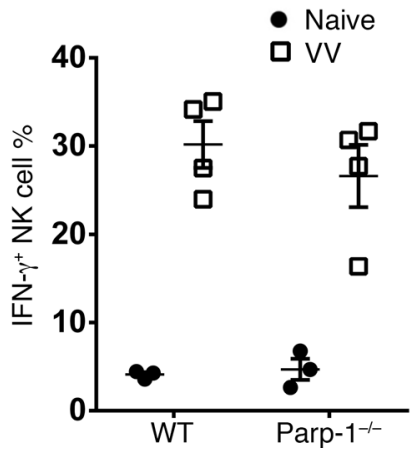

B
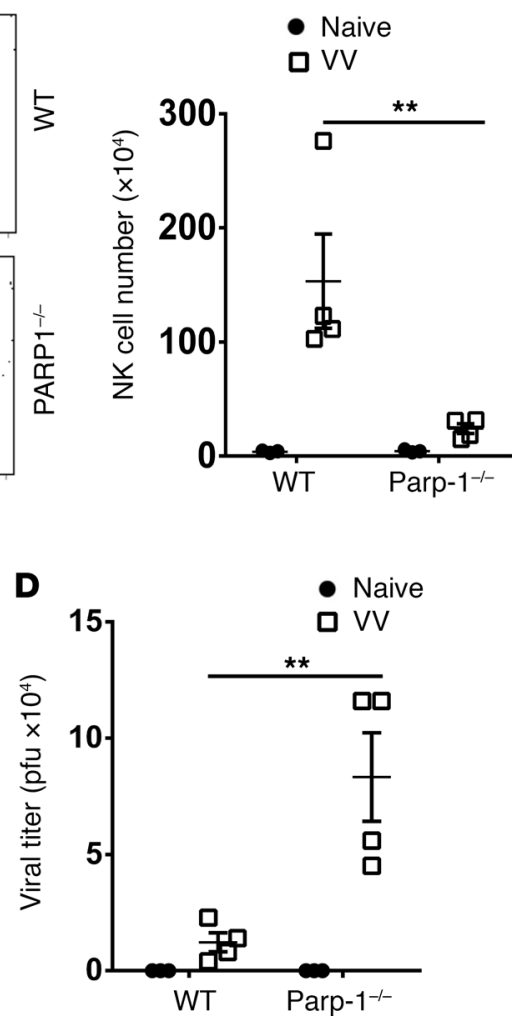

Figure 2. Defective NK cell recruitment and viral clearance in Parp-1/- mice. WT or Parp-1/- mice were infected with VV $\left(5 \times 10^{6} \mathrm{PFU}\right.$, i.p.) and some mice were left uninfected as controls (Naive). Three days later, peritoneal fluid was harvested and assayed for DX5 ${ }^{+} \mathrm{CD}^{-}$NK cells by flow cytometry. (A) FACS plots showing percentages of DX5 ${ }^{+} \mathrm{CD}^{-} \mathrm{NK}$ cells in the peritoneal fluids. (B) The mean absolute NK cell numbers \pm SD are shown $(n=3)$. (C) IFN- $\gamma$ production was measured intracellularly and the mean percentages of IFN- $\gamma^{+}$NK cells are shown $(n=3)$. (D) The viral titer in peritoneal fluid was measured by the standard plaque assay and the mean viral titers \pm SD are shown $(n=4) .{ }^{*} P<0.01,2$-tailed Student's $t$ test.

macrophages in response to VV infection. NF-kB is composed of dimeric subunits of p65 and p50 (26).

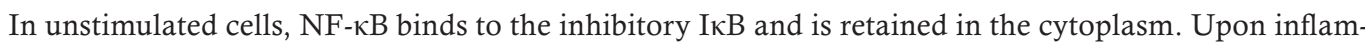
matory stimulation, IкB is phosphorylated by IкB kinases (IKKs) and degraded by the ubiquitin and proteasome system, which causes translocation of NF- $\mathrm{kB}$ to the nucleus, where it binds to its response DNA elements and activates the transcription of a number of genes, including inflammatory genes. Indeed, we showed that VV infection promoted NF-kB translocation to the nucleus in WT macrophages (Figure 7A) compared with the uninfected controls, whereas in Parp-1/-1 macrophages NF-kB nuclear translocation was reduced (Figure 7A), suggesting that PARP-1 promotes translocation of NF- $\mathrm{kB}$ to the nucleus upon VV infection. In addition, we found that the binding of NF- $\mathrm{BB}$ to its response DNA is inhibited in the absence of PARP-1 (Figure 7B), suggesting PARP-1 also promotes binding to its promoter sequences upon nuclear translocation. Thus, PARP-1 functions as a regulator of NF- $\mathrm{kB}$ by promoting its nuclear translocation and binding to its response sequences in macrophages.

\section{Discussion}

In this study, we revealed a critical role for PARP-1 in NK cell recruitment to the site of VV infection and viral control in vivo. We further demonstrated that PARP-1 promotes the production of CCL2 and that the CCL2-CCR2 axis is essential for NK cell recruitment to the infection site. In addition, we showed that peritoneal macrophages are mainly responsible for PARP-1-dependent CCL2 secretion. Furthermore, PARP-1 functions as a regulator of NF-KB by promoting its nuclear translocation and binding to its response sequences in macrophages upon VV infection.

Although the recruitment of activated NK cells to the site of VV infection and its role in viral control have been well recognized (6-9), what controls NK recruitment to the site of VV infection 
A

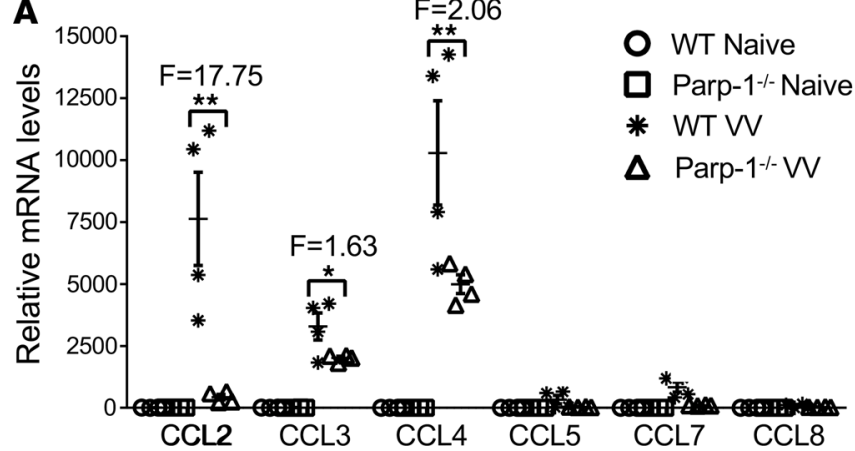

B

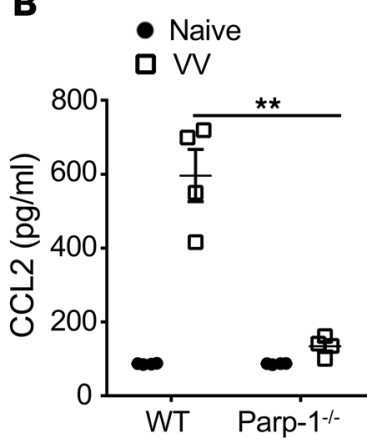

Figure 3. The production of CCL2 in response to VV infection is diminished in Parp-1/- mice. WT or Parp-1/- mice were infected with VV $\left(5 \times 10^{6} \mathrm{PFU}\right.$, i.p.) and some mice were left uninfected as controls (Naive). (A) 12 hours after infection, intraperitoneal cells were collected and assayed for CCL2, CCL3, CCL4, CCL5, CCL7, and CCL8 mRNA levels by real-time quantitative PCR. Relative mRNA levels were normalized to $\beta$-actin RNA within each sample. Data represent mean relative mRNA levels \pm SD $(n=3)$. Folds (F) of change in samples from VV-infected WT (WT VV) over Parp1 ${ }^{-1-}\left(\right.$ Parp1 $1^{-1-}$ VV) mice are shown. (B) 24 hours after infection, peritoneal fluid was harvested and measured for CCL2 production by the colorimetric beads assay. Data represent mean CCL2 concentrations $(\mathrm{pg} / \mathrm{ml}) \pm \mathrm{SD}(n=3) .{ }^{*} P<0.05,{ }^{* *} P<0.01$, 2-tailed Student's $t$ test.

remained unknown. Here we showed that the CCL2-CCR2 axis is critical for NK cell migration to the peritoneal cavity, where VV infection occurs. Our observation is consistent with the role of CCR2 in NK cell migration to the liver of MCMV-infected mice $(12,13)$ and to the lung during invasive aspergillosis (28). This is also in line with the role of CCL2 in NK cell recruitment to the lungs during modified VV ankara (MVA) infection (29). In addition to CCL2, we found that the expression of CCL3 (a chemokine for CCR1 and CCR5) (30) and CCL4 (a chemokine for CCR5) (30) was also significantly induced in response to VV infection. Indeed, CCR5 and CXCR3 are utilized for NK cell migration to the CNS in a model of HSV infection (14) and to the liver of dengue virus-infected mice (15), respectively, whereas CCR1 is involved in NK cell trafficking to the liver in concanavalin A-induced (Con A-induced) hepatitis (31). Thus, it would be important to determine whether CCL3 or CCL4 is also critical for NK cell migration in response to VV infection in future studies.

It has been well documented that inflammatory stimuli elicit the production of various chemokines that contribute to NK cell migration to inflammatory sites (11). However, the underlying molecular and cellular mechanisms responsible for chemokine production in response to inflammatory stimuli
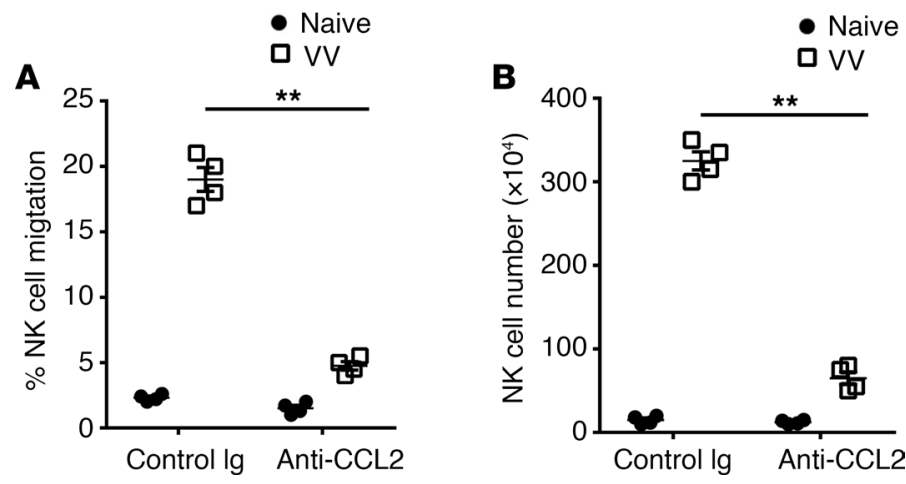

Figure 4. CCL2 is responsible for NK cell recruitment to the site of VV infection. (A) C57B/L6 mice were infected with VV $\left(5 \times 10^{6} \mathrm{PFU}\right.$, i.p.) or left uninfected (Naive). 72 hours later, the peritoneal CD3-DX5+ NK cells and the peritoneal fluid were collected for in vitro NK cell migration assay using the Transwell chamber. The peritoneal fluid was mixed with a neutralizing anti-CCL2 antibody $(2 \mu \mathrm{g} / \mathrm{ml})$ or a control lg and added in the lower chamber, and NK cells were added to the upper chamber. After 5 hours incubation at $37^{\circ} \mathrm{C}$, the number of NK cells migrated to the lower chamber was measured. The data represent \%NK cells \pm SD $(n=4)$ migrated to the lower chamber over total input NK cells on the upper chamber. (B) C57BL/ 6 mice were treated with anti-CCL2-neutralizing antibody (20 $\mu \mathrm{g}$ per mouse, daily beginning day -1) or a control Ig. On day 0, mice were infected with VV (5 × $10^{6} \mathrm{PFU}$, i.p.) or left uninfected (naive). Three days later, peritoneal fluid was harvested and assayed for DX5 ${ }^{+}$CD3 ${ }^{-}$NK cells by flow cytometry. The mean absolute NK cell numbers \pm SD are shown $(n=3) .{ }^{* *} P<0.01,2$-tailed Student's $t$ test. 
A
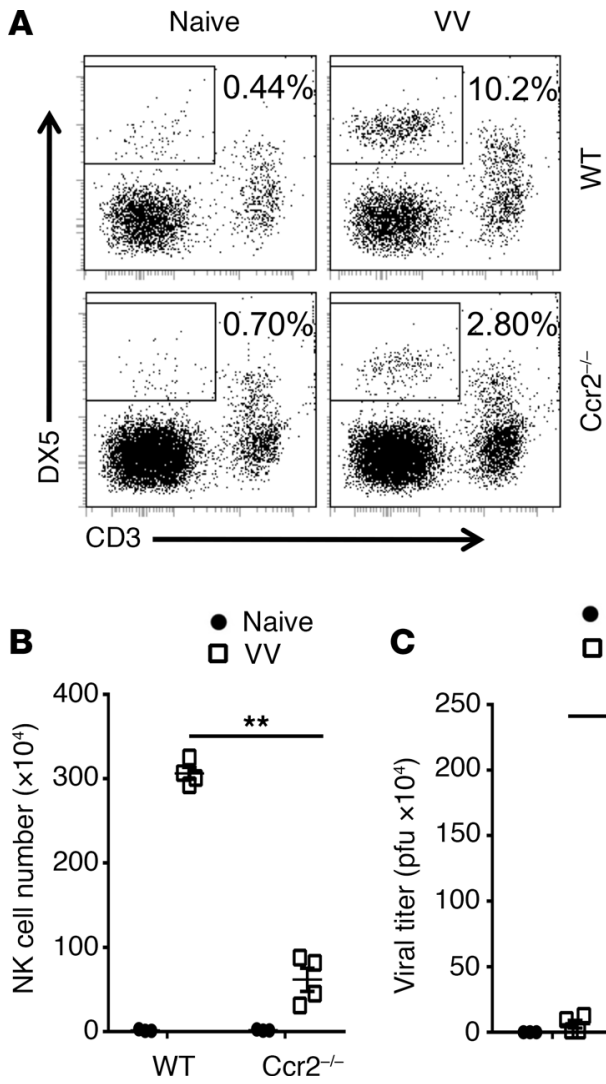

々

C

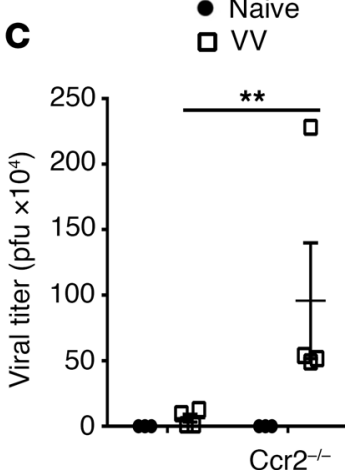

Figure 5. CCR2 mediates NK cell migration in response to VV infection. WT or $\mathrm{Ccr}^{-/-}$mice were infected with VV $\left(5 \times 10^{6} \mathrm{PFU}\right.$, i.p. $)$ or left uninfected as controls (Naive). Three days later, peritoneal fluid was harvested and assayed for DX5 ${ }^{+} \mathrm{CD}^{-}{ }^{-}$NK cells by flow cytometry. (A) FACS plots showing percentages of DX5+CD3- NK cells in the peritoneal fluids. (B) The mean absolute NK cell numbers \pm SD are shown $(n=3)$. (C) The viral titer in peritoneal fluid was measured by the standard plaque assay and the mean viral titers \pm SD are shown $(n=4) .{ }^{*} P<0.01$, 2 -tailed Student's $t$ test.

remained largely undefined. In a model of VV infection, we revealed that PARP-1, a DNA damage sensor and mediator of inflammation, plays a critical role in the recruitment of NK cells via the production of CCL2 by infiltrating macrophages. This is accomplished by promoting NF- $\mathrm{kB}$ nuclear translocation and binding to its response sequences in macrophages. It remains to be determined whether CCL2 production is regulated by NF-kB directly or directly via cytokines such as TNF- $\alpha$, which has been shown to upregulate the expression of CCL2 in sensory neurons (32). Furthermore, as we have shown that type I IFNs are produced during VV infection $(8,10)$, the type I IFN pathway could also contribute to the CCL2 expression as shown in a model of MVA infection (29). Although PARP-1 has been implicated in the development of inflammation in a variety of models (19-22) and in the suppression of regulatory $\mathrm{T}$ cells $(23,24)$, our results support, for the first time to our knowledge, a critical role for PARP-1 in NK cell migration to the site of inflammation. Whether PARP-1 also regulates the migration of other inflammatory cells such as T cells remains to be determined in the future. Besides PARP-1, PARP-2 may also be involved in the regulation of inflammatory processes (33). Thus, it will be also important to investigate the role of other members of the PARP family in the regulation of inflammatory cell migration.

PARP-1 is activated by binding to damaged DNA (17). How does VV infection in macrophages lead to DNA damage that triggers the activation of PARP-1? Macrophages play a crucial role in the control of invading pathogens through the production of reactive oxygen species (ROS) or reactive nitrogen species (RNS) (34), which can damage cellular DNA $(35,36)$. In addition, the generation of ROS and RNS could further activate the redox-sensitive transcription factor, NF- $\kappa \mathrm{B}$, a key regulator of iNOS and cyclooxygenase-2 (COX-2), leading to production of more ROS and RNS (37-39). Thus, future studies are needed to further define mechanisms underlying DNA damage and activation of PARP-1 in response to viral infections. Similarly, although our results suggest that peritoneal macrophages are responsible for PARP1-dependent CCL2 production in the peritoneal cavity, it remains to be determined whether tissue resident macrophages are also involved in the production of CCL2 upon viral infection.

In summary, we have provided evidence to support a critical role for PARP-1 in NK cell migration to the site of VV infection and viral control in vivo through induction of CCL2 by macrophages. Mechanistically, PARP-1 functions as a regulator of NF- $\mathrm{KB}$ by promoting its nuclear translocation and binding to its response 
A
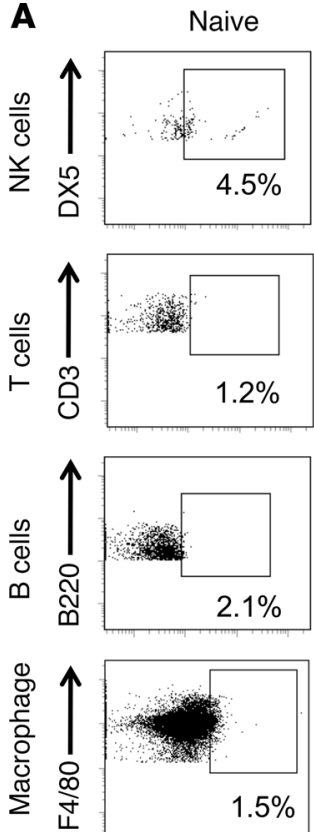

CCL2
VV
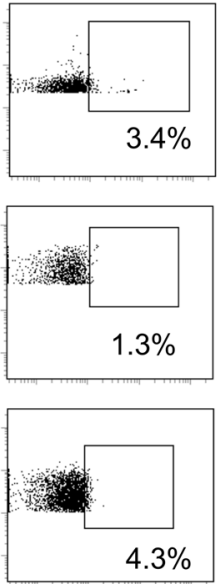

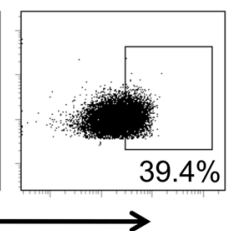

B

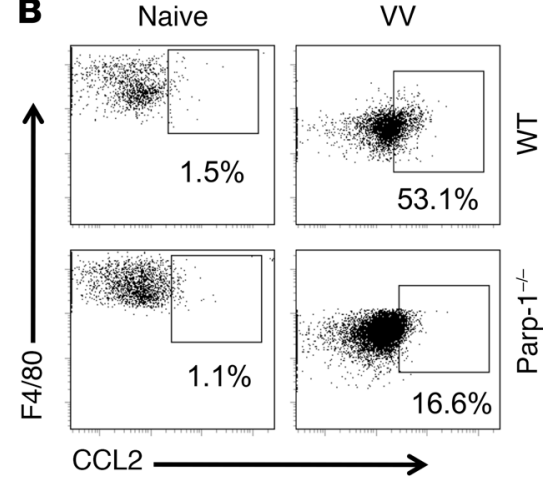

C

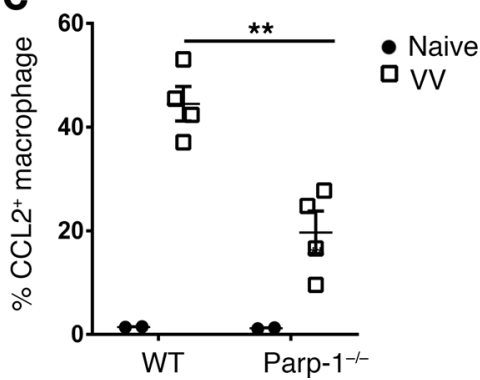

Figure 6. Peritoneal macrophages are the major producer of PARP-1 dependent CCL2. (A) C57B/L6 mice were infected with VV $\left(5 \times 10^{6} \mathrm{PFU}\right.$, i.p.) or left uninfected (Naive). Three days after infection, CCL2 production in NK, T, and B cells, as well as macrophages, from peritoneal fluid, was measured by intracellular staining. The data represent percentages of CCL2 ${ }^{+}$cells among their respective cell population. (B and C) WT or Parp-1/- mice were infected with VV $\left(5 \times 10^{6}\right.$ PFU, i.p.) or left uninfected as controls (Naive). Three days later, CCL2 production by the peritoneal macrophages was measured by intracellular staining. FACS plots showing percentages of $\mathrm{CCL}^{+}$cells among total macrophages (B). Mean percentages $\pm \mathrm{SD}$ of $\mathrm{CCL}^{+}$cells $(n=3)(\mathbf{C}) .{ }^{* *} P<0.01,2$-tailed Student's $t$ test.

sequences in macrophages upon VV infection. Taken together, our results reveal a previously unknown role for PARP-1-dependent CCL2 production in NK cell migration in response to viral infection and may provide important insights into the design of effective NK cell-based therapies for viral infections and cancer.

\section{Methods}

Mice. Parp- $1^{-/}, \mathrm{Ccr2}^{-/-}$, and CD45.1 C57BL/6 mice were purchased from The Jackson laboratory. C57BL/ 6 mice were from Charles River Laboratories. Parp- $1^{-/-}$mice are viable, fertile, normal in size, and do not display any gross physical or behavioral abnormalities, nor are they susceptible to infections. Mice used for experiments were 6-8 weeks old.

PARP-1 inhibitor. PARP-1 inhibitor (AG14361) was purchased from Selleckchem and dissolved in DMSO at a stock concentration of $40 \mathrm{mM}$ and stored at $-20^{\circ} \mathrm{C}$. AG14361 was added to cell culture at a final concentration of $20 \mu \mathrm{M}$. For in vivo studies, $10 \mathrm{mg} / \mathrm{kg}$ AG14361 was injected into mice intravenously.

$V V$. The Western Reserve strain of VV was grown in TK-143B cells and purified by centrifugation through a $35 \%$ sucrose cushion as described previously (40). The titer of virus was determined by plaque assay on TK-143B cells and stored at $-80^{\circ} \mathrm{C}$. For in vivo studies, $5 \times 10^{6} \mathrm{PFU}$ of live VV in $0.05 \mathrm{~mL}$ of 1 $\mathrm{mM}$ Tris- $\mathrm{HCl}(\mathrm{pH} 9.5)$ was injected into mice intraperitoneally as described previously (40). For in vitro macrophage stimulations, VV were used at a multiplicity of infection (MOI) of 0.5 .

Antibodies and flow cytometry. Phycoerythrin-conjugated (PE-conjugated) anti-DX5, PE-Cy5-conjugated anti-CD3 $\varepsilon$, allophycocyanin-conjugated (APC-conjugated) anti-B220, APC-conjugated antiCD11b, APC-conjugated anti-CD45.1, FITC-conjugated anti-CD45.2, FITC-conjugated anti-CCL2 were purchased from BD Biosciences. APC-conjugated anti-IFN- $\gamma$ and PE-conjugated anti-F4/80 were purchased from BioLegend. To assess production of IFN- $\gamma$ and CCL2, peritoneal cavity cells were incubated with $100 \mathrm{ng} / \mathrm{ml}$ PMA and ionomycin and $25 \mathrm{ng} / \mathrm{ml}$ Brefeldin A (BD Biosciences) for 4 hours at $37^{\circ} \mathrm{C}$. Intracellular staining was performed as previously described (10). Briefly, the cells were first stained with anti-DX5, - CD3, or anti-F4/80, -CD11b followed by fixation and permeabilization with 


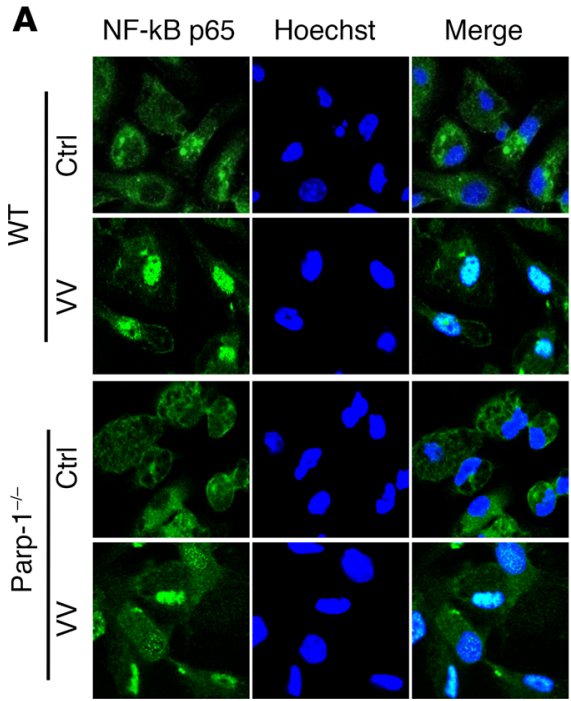

B

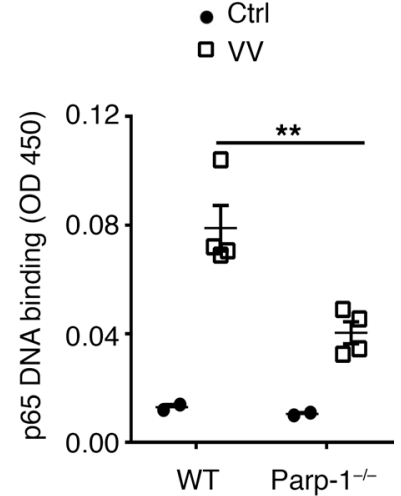

Figure 7. PARP-1 promotes NF- $\mathrm{BB}$ nuclear translocation and DNA binding in microphages. Peritoneal macrophages from WT or Parp-1-1- mice were infected with VV (MOI = 0.5) for 1 hour or left uninfected as a control (Ctrl). (A) After staining with anti-NF-kB-p65 (green) and nuclear (blue) staining, representative confocal microscopy images are shown. Original magnification, $\times 200$. (B) NF-kB containing nuclear extract was measured for the ability to bind to a specific double-stranded DNA (dsDNA) sequence containing the NF- $\mathrm{kB}-\mathrm{p} 65$ response element by a colorimetric assay. Data represent mean OD450 readouts $\pm \operatorname{SD}(n=3) .{ }^{* *} P<0.01$, 2-tailed Student's $t$ test.

Cytofix/Cytoperm (BD Biosciences). The cells were subsequently stained intracellularly with anti-IFN- $\gamma$ or CCL2 and then subjected to flow cytometry analysis with FACSCanto (BD Biosciences). FACSDiVA software (BD Biosciences) was used for data analysis.

Quantitative real-time PCR. Total RNA was prepared from peritoneal cells using TriZol reagent (Thermo Fisher). cDNA was synthesized with GoScript Reverse Transcriptase and Oligo(dT)15 Primer(Promega). Subsequently, the gene expression was examined by using Bio-Rad CFX96 System with iTaq Universal SYBR Green Supermix Real-Time PCR kit (Bio-Rad). The expression of each gene was normalized to $\beta$-actin reference and compared. The primers for CCL2, CCL3, CCL4, CCL5, CCL7, and CCL8 were CCL2 forward: 5'-GATCCCAATGAGTAGGCTGG-3'; CCL2 reverse: 5'-CGGGTCAACTTCACATTCAAAG-3'; CCL3 forward: 5'-ACACCAGAAGGATACAAGCAG-3'; CCL3 reverse: 5'-CGATGAATTGGCGTGGAATC-3'; CCL4 forward: 5'-AAACCTAACCCCGAGCAAC-3'; CCL4 reverse: 5'-CTGTCTGCCTCTTTTGGTCA-3'; CCL5 forward: 5'-CCCACGTCAAGGAGTATTTCTAC-3'; CCL5 reverse: 5'-CTAGGACTAGAGCAAGCGATG-3'; CCL 7 forward: 5'-TCTCTCACTCTCTTTCTCCACC-3'; CCL7 reverse: 5'-GGTGATCCTTCTGTAGCTCTTG-3'; CCL8 forward: 5'-CAGATAAGGCTCCAGTCACC-3'; and CCL8 reverse: 5'-GCTGTCTCTTAACTCAGGTGTG-3'.

Chemokine and cytokine analysis. The level of chemokines and cytokines in the peritoneal lavage fluids and the supernatants of macrophage culture was measured by Cytometric Bead Array (CBA) assay using the Mouse Inflammation Kit according to the manufacturer's instructions (BD Biosciences).

NK cell migration assay. Mice were infected with VV and 3 days later, the peritoneal NK cells and the peritoneal fluid were collected for NK cell migration using the Transwell migration chamber (pore size: 5 $\mu \mathrm{m})$. The peritoneal fluid from macrophages was added to the lower chamber as chemoattractant, and NK cells were added to the upper chamber. After 5 hours at $37^{\circ} \mathrm{C}$ incubation, the number of NK cells in the lower chamber was determined by FACS (BD Biosciences), and the percentage of NK cells that migrated to the lower chamber over the total input of NK cells into the upper chamber was calculated.

In vitro and in vivo antibody blocking assay. For in vitro antibody blocking, the peritoneal fluid was mixed with a neutralizing goat anti-mouse CCL2 antibody ( $2 \mu \mathrm{g} / \mathrm{ml}$, R\&D Systems) or a control Ig and used for the NK cell migration assay. For in vivo antibody blocking, mice were treated with anti-CCL2neutralizing antibody $(20 \mu \mathrm{g}$ per mouse, daily beginning day -1$)$ or a control Ig. On day 0 , mice were infected with VV $\left(5 \times 10^{6} \mathrm{PFU}\right.$, intraperitoneally). Three days later, peritoneal fluid was harvested and assayed for $\mathrm{DX} 5^{+} \mathrm{CD}^{-} \mathrm{NK}$ cells by flow cytometry. 


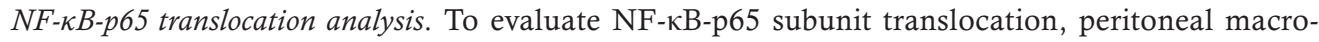
phages were stimulated with VV at MOI of 0.5 for 1 hour and then incubated with a 1:50 mouse monoclonal anti-NF-kB-p65 (Santa Cruz Biotech) at $4^{\circ} \mathrm{C}$ for 18 hours, followed by staining with Alexa Fluor 488-conjugated anti-mouse IgG (Invitrogen) at a 1:200 dilution at room temperature for 1 hour. Cells were subsequently incubated with Hoechst $33342(0.2 \mu \mathrm{g} / \mathrm{ml})$ for 10 minutes to stain the nucleus. Confocal microscopy of macrophages was taken with a Leica SP5 confocal microscope. The lasers used to excite Alexa Fluor 488 and Hoechst were $488 \mathrm{~nm}$ and $405 \mathrm{~nm}$, respectively. Leica LAS AF software was used for image analysis.

Assay of $N F-\kappa B-D N A$-binding activity. $6 \times 10^{6}$ macrophages were seeded on a $100-\mathrm{mm}$ plate. After treatment and VV stimulation, cells were washed with PBS twice and then harvested and counted. Cells suspended in $100 \mu \mathrm{L}$ Pre-Extraction Buffer per $10^{6}$ cells were used for nuclear protein extraction. Cell nuclear extract was utilized to investigate NF-кB-p65 DNA-binding with an Abcam kit. As described in the manufacturer's manual, a specific double-stranded DNA (dsDNA) sequence containing the NF- $\mathrm{B}$ response element was coated onto the bottom of wells of a 96-well plate. NF- $\kappa B$ contained within nuclear extract binds specifically to the NF- $\mathrm{kB}$ response element and was detected by addition of a specific primary antibody directed against NF- $\mathrm{kB}-\mathrm{p} 65$. A secondary antibody conjugated to HRP was added to provide a sensitive colorimetric readout at $450 \mathrm{~nm}$.

Statistics. Results are expressed as mean \pm SEM. Comparison between groups was performed by 2 -tailed Student's $t$ test. All statistical analyses were performed with SPSS19.0 software (IBM). $P$ values of less than 0.05 are considered to be significant.

Study approval. All experiments involving the use of mice followed NIH guidelines and were done in accordance with protocols approved by the Institutional Animal Care and Use Committee of Duke University.

\section{Author contributions}

QS and HF designed and performed the study, collected and analyzed data, and prepared the paper; HF performed select experiments and collected and analyzed data; and XH and YY designed research, analyzed data, and wrote the paper.

\section{Acknowledgments}

We thank Yuqing Yuan for the technical support. This work was supported by NIH grants CA136934, CA186973, and CA193167 (to YY).

Address correspondence to: Xiaopei Huang, Department of Medicine, Duke University Medical Center, Box 103005, Durham, North Carolina 27710, USA. Phone: 919.684.9511; Email: huang027@mc.duke.edu. Or to: Yiping Yang, Department of Medicine, Duke University Medical Center, Box 103005, Durham, North Carolina 27710, USA. Phone: 919.668.0932; Email: yang0029@mc.duke.edu.

1. French AR, Yokoyama WM. Natural killer cells and viral infections. Curr Opin Immunol. 2003;15(1):45-51.

2. Lee SH, Miyagi T, Biron CA. Keeping NK cells in highly regulated antiviral warfare. Trends Immunol. 2007;28(6):252-259

3. Lanier LL. Up on the tightrope: natural killer cell activation and inhibition. Nat Immunol. 2008;9(5):495-502.

4. Biron CA, Byron KS, Sullivan JL. Severe herpesvirus infections in an adolescent without natural killer cells. $N$ Engl J Med. 1989;320(26):1731-1735.

5. Fenner F, Henderson D, Arita I, Jezek Z, Ladnyi I; World Health Organization. Smallpox and its eradication. Geneva, Switzerland: World Health Organization; 1988.

6. Bukowski JF, Woda BA, Habu S, Okumura K, Welsh RM. Natural killer cell depletion enhances virus synthesis and virus-induced hepatitis in vivo. J Immunol. 1983;131(3):1531-1538.

7. Natuk RJ, Welsh RM. Accumulation and chemotaxis of natural killer/large granular lymphocytes at sites of virus replication. J Immunol. 1987;138(3):877-883.

8. Martinez J, Huang X, Yang Y. Direct action of type I IFN on NK cells is required for their activation in response to vaccinia viral infection in vivo. J Immunol. 2008;180(3):1592-1597.

9. Martinez J, Huang X, Yang Y. Toll-like receptor 8-mediated activation of murine plasmacytoid dendritic cells by vaccinia viral DNA. Proc Natl Acad Sci U S A. 2010;107(14):6442-6447.

10. Zhu J, Martinez J, Huang X, Yang Y. Innate immunity against vaccinia virus is mediated by TLR2 and requires TLR-independent production of IFN- $\beta$. Blood. 2007;109(2):619-625.

11. Grégoire C, Chasson L, Luci C, et al. The trafficking of natural killer cells. Immunol Rev. 2007;220:169-182.

12. Hokeness KL, Kuziel WA, Biron CA, Salazar-Mather TP. Monocyte chemoattractant protein-1 and CCR2 interactions are required for IFN- $\alpha / \beta$-induced inflammatory responses and antiviral defense in liver. J Immunol. 2005;174(3):1549-1556. 
13. Salazar-Mather TP, Orange JS, Biron CA. Early murine cytomegalovirus (MCMV) infection induces liver natural killer (NK) cell inflammation and protection through macrophage inflammatory protein 1alpha (MIP-1alpha)-dependent pathways. $J$ Exp Med. 1998;187(1):1-14.

14. Thapa M, Kuziel WA, Carr DJ. Susceptibility of CCR5-deficient mice to genital herpes simplex virus type 2 is linked to NK cell mobilization. J Virol. 2007;81(8):3704-3713.

15. Hsieh MF, Lai SL, Chen JP, et al. Both CXCR3 and CXCL10/IFN-inducible protein 10 are required for resistance to primary infection by dengue virus. J Immunol. 2006;177(3):1855-1863.

16. Amé JC, Spenlehauer C, de Murcia G. The PARP superfamily. Bioessays. 2004;26(8):882-893.

17. Kraus WL. Transcriptional control by PARP-1: chromatin modulation, enhancer-binding, coregulation, and insulation. Curr Opin Cell Biol. 2008;20(3):294-302.

18. Kim MY, Zhang T, Kraus WL. Poly(ADP-ribosyl)ation by PARP-1: 'PAR-laying' NAD' into a nuclear signal. Genes Dev. 2005;19(17):1951-1967.

19. Scott GS, Hake P, Kean RB, Virág L, Szabó C, Hooper DC. Role of poly(ADP-ribose) synthetase activation in the development of experimental allergic encephalomyelitis. J Neuroimmunol. 2001;117(1-2):78-86.

20. Zingarelli B, Szabó C, Salzman AL. Blockade of poly(ADP-ribose) synthetase inhibits neutrophil recruitment, oxidant generation, and mucosal injury in murine colitis. Gastroenterology. 1999;116(2):335-345.

21. Oliver FJ, et al. Resistance to endotoxic shock as a consequence of defective NF-kappaB activation in poly (ADP-ribose) polymerase-1 deficient mice. EMBO J. 1999;18(16):4446-4454.

22. Liaudet L, et al. Protection against hemorrhagic shock in mice genetically deficient in poly(ADP-ribose)polymerase. Proc Natl Acad Sci U S A. 2000;97(18):10203-10208.

23. Nasta F, Laudisi F, Sambucci M, Rosado MM, Pioli C. Increased Foxp3+ regulatory T cells in poly(ADP-Ribose) polymerase-1 deficiency. J Immunol. 2010;184(7):3470-3477.

24. Zhang P, et al. PARP-1 controls immunosuppressive function of regulatory T cells by destabilizing Foxp3. PLoS One. 2013;8(8):e71590

25. Veuger SJ, Hunter JE, Durkacz BW. Ionizing radiation-induced NF-kappaB activation requires PARP-1 function to confer radioresistance. Oncogene. 2009;28(6):832-842.

26. Hayden MS, Ghosh S. Shared principles in NF-кB signaling. Cell. 2008;132(3):344-362.

27. Bai P, Virág L. Role of poly(ADP-ribose) polymerases in the regulation of inflammatory processes. FEBS Lett. 2012;586(21):3771-3777.

28. Morrison BE, Park SJ, Mooney JM, Mehrad B. Chemokine-mediated recruitment of NK cells is a critical host defense mechanism in invasive aspergillosis. J Clin Invest. 2003;112(12):1862-1870.

29. Lehmann MH, Torres-Domínguez LE, Price PJ, Brandmüller C, Kirschning CJ, Sutter G. CCL2 expression is mediated by type I IFN receptor and recruits NK and T cells to the lung during MVA infection. J Leukoc Biol. 2016;99(6):1057-1064.

30. Deshmane SL, Kremlev S, Amini S, Sawaya BE. Monocyte chemoattractant protein-1 (MCP-1): an overview. J Interferon Cytokine Res. 2009;29(6):313-326.

31. Wald O, et al. IFN-gamma acts on T cells to induce NK cell mobilization and accumulation in target organs. J Immunol. 2006;176(8):4716-4729.

32. Jung H, Miller RJ. Activation of the nuclear factor of activated T-cells (NFAT) mediates upregulation of CCR2 chemokine receptors in dorsal root ganglion (DRG) neurons: a possible mechanism for activity-dependent transcription in DRG neurons in association with neuropathic pain. Mol Cell Neurosci. 2008;37(1):170-177.

33. Szántó M, et al. Poly(ADP-ribose) polymerase-2: emerging transcriptional roles of a DNA-repair protein. Cell Mol Life Sci. 2012;69(24):4079-4092.

34. Medzhitov R. Inflammation 2010: new adventures of an old flame. Cell. 2010;140(6):771-776.

35. Mangerich A, et al. Infection-induced colitis in mice causes dynamic and tissue-specific changes in stress response and DNA damage leading to colon cancer. Proc Natl Acad Sci U S A. 2012;109(27):E1820-E1829.

36. Lonkar P, Dedon PC. Reactive species and DNA damage in chronic inflammation: reconciling chemical mechanisms and biological fates. Int J Cancer. 2011;128(9):1999-2009.

37. Szabó C, Ischiropoulos H, Radi R. Peroxynitrite: biochemistry, pathophysiology and development of therapeutics. Nat Rev Drug Discov. 2007;6(8):662-680.

38. Mungrue IN, et al. Cardiomyocyte overexpression of iNOS in mice results in peroxynitrite generation, heart block, and sudden death. J Clin Invest. 2002;109(6):735-743

39. Lin Y, Tang X, Zhu Y, Shu T, Han X. Identification of PARP-1 as one of the transcription factors binding to the repressor element in the promoter region of COX-2. Arch Biochem Biophys. 2011;505(1):123-129.

40. Yang Y, Huang CT, Huang X, Pardoll DM. Persistent Toll-like receptor signals are required for reversal of regulatory T cell-mediated CD8 tolerance. Nat Immunol. 2004;5(5):508-515. 